\title{
A STONG-HATTORI SPECTRAL SEQUENCE
}

\author{
BY \\ DAVID COPELAND JOHNSON
}

\begin{abstract}
Let $G_{*}()$ be the Adams summand of connective $K$-theory localized at the prime $p$. Let $B P_{*}()$ be Brown-Peterson homology for that prime. A spectral sequence is constructed with $E^{2}$ term determined by $G_{*}(X)$ and whose $E^{\infty}$ terms give the quotients of a filtration of $B P_{*}(X)$ where $X$ is a connected spectrum. A torsion property of the differentials implies the Stong-Hattori theorem.
\end{abstract}

1. Introduction. $M U$ is the Thom spectrum which represents complex bordism, $M U_{*}()$, and $b u$ is the connective unitary spectrum whose associated homology theory is complex connective $K$-theory, $k_{*}() . b u$ is a ring spectrum with unit $i: S^{0} \rightarrow b u$. One formulation of the Stong-Hattori theorem ([12], [23]) is: if $X$ is a complex with free abelian integral homology, then

$$
\operatorname{sh}_{X}: M U_{*}(X) \rightarrow k_{*}(X \wedge M U)
$$

is a split monomorphism where $\mathrm{sh}_{X}$ is induced by the composition

$$
\begin{gathered}
M U \wedge X \simeq M U \wedge S^{0} \wedge X \stackrel{M U \wedge i \wedge X}{\longrightarrow} M U \wedge b u \wedge X \\
\stackrel{\text { interchange }}{\longrightarrow} b u \wedge X \wedge M U .
\end{gathered}
$$

Of course, $\mathrm{sh}_{X}$ is an analog of the Hurewicz homomorphism

$$
h_{X}: M U_{*}(X) \rightarrow H_{*}(X \wedge M U ; Z)
$$

constructed from the unit $j: S^{0} \rightarrow K Z$, of the (integral) Eilenberg-Mac Lane spectrum. If $\left\{E_{p, q}^{r}(X), d^{r}(X)\right\}$ is the Atiyah-Hirzebruch-Dold spectral sequence

$$
E_{s, t}^{2}(X) \cong H_{s}\left(X ; M U_{t}\right) \Rightarrow M U_{*}(X),
$$

then one can prove $h_{X}$ is monic whenever

$$
E_{*, *}^{\infty}(X \wedge j): E_{*, *}^{\infty}\left(X \wedge S^{0}\right) \rightarrow E_{*, *}^{\infty}(X \wedge K Z)
$$

is monic. In particular, this is true when $E_{*, *}^{2}(X)$ is torsion-free. The object of this paper is to develop a spectral sequence whose relation to $\operatorname{sh}_{X}$ is analogous to the above relation of the Atiyah-Hirzebruch-Dold spectral sequence to $h_{x}$.

Received by the editors May 16, 1972.

AMS (MOS) subject classifications (1970). Primary 55B20, 55H25, 57D90.

Key words and phrases. Atiyah-Hirzebruch-Dold spectral sequence, Stong-Hattori theorem, complex bordism, Brown-Peterson spectrum, connective $k$-theory. 
We shall be working in Boardman's stable category as described in [24]. A connected spectrum will mean an object in Boardman's category which is $n$ connected for some (possibly negative) integer $n$. $S$ denotes Boardman's suspension-translation functor. $S^{0}$ is the $((-1))$-connected) sphere spectrum and * is the trivial (base point) spectrum. By homology theory, we shall mean a reduced generalized homology theory with countable coefficient graded group. Recall that homology theories defined on finite complexes extend to ones defined on spectra $[24,13.6]$.

Fix a prime $p$. We shall follow the algebraist's notation in letting $Z_{(p)}$ be the localization of $Z$ at the prime ideal $(p)$. So $Z_{(p)}$, of ten called $Q_{p}$, is the subring of the rationals of form $a / b, \operatorname{gcd}(a, b)=1$ and $\operatorname{gcd}(b, p)=1$. We write $Z / p Z$ (instead of $Z_{p}$ ) for the cyclic group of order $p$. Since $Z_{(p)}$ is a flat abelian group, $M U_{(p) *}(X)=M U_{*}(X) \otimes Z_{(p)}$ and $k_{(p) *}(X)=k_{*}(X) \otimes Z_{(p)}$ define homology theories. Quillen has showed that there is a multiplicative idempotent $\varepsilon$ $\in M U_{(p)}^{0}\left(M U_{(p)}\right)$ which splits $M U_{(p) *}()$ into a direct sum of shifted copies of $B P_{*}()=\varepsilon\left(M U_{(p) *}()\right)[2]$. Here $B P_{*}()$ is the Brown-Peterson homology theory induced by the Brown-Peterson spectrum $B P$ [6].

$$
B P_{*}=\pi_{*}(B P) \cong Z_{(p)}\left[v_{1}, v_{2}, \ldots, v_{s}, \ldots\right]
$$

with degree $v_{s}=2_{p}^{s}-2$. In this paper, we shall assume that it is clear that constructions in complex bordism theory can be copied in Brown-Peterson homology. Note that if hom $\operatorname{dim}_{R} M$ means the projective dimension of $M$ as an $R$-module, then

$$
\text { hom } \begin{aligned}
\operatorname{dim}_{M U_{*}} M U_{*}(X) & \geq \text { hom } \operatorname{dim}_{M U_{(p) *}} M U_{(p) *}(X) \\
& =\text { hom } \operatorname{dim}_{B P_{*}} B P_{*}(X) .
\end{aligned}
$$

Also, Adams has given a splitting of $k_{(p) *}$ ( ) into a direct sum of multiplicative theories $G_{*}()$ induced by the ring spectrum $G \cdot \pi_{*}(G) \cong Z_{(p)}[u]$ with degree $u=2 p-2([1],[3])$.

$\S 2$ gives a setting for spectral sequences of the Atiyah-Hirzebruch-Dold type relating two generalized homology theories. Also there is a definition of $\langle u\rangle$ torsion in a $Z_{(p)}[u]$-module and a definition of $\langle u\rangle$-torsion valued differentials in a spectral sequence whose $E^{2}$ term is a $Z_{(p)}[u]$-module (but whose differentials need not be $Z_{(p)}[u]$ homomorphisms). The third section reviews necessary properties of the spectra $B P$ and $G$. The author thanks A. Liulevicius and $R$. Zahler for their information on $B P_{*}$ generators and $B P$ operators which allows for an economical discussion in this section. The fourth section constructs the spectral sequence which is described by (4.3).

Theorem 4.3. For any connected spectrum $X$, there is a natural spectral sequence of the Atiyah-Hirzebruch-Dold type,

$$
E_{n-q, *}^{2}(X) \cong G_{n-q}(X) \otimes Z_{(p)}\left[v_{2}, v_{3}, \ldots\right] \Rightarrow B P_{*}(X) .
$$

The differentials of this spectral sequence are $\langle u\rangle$-torsion valued. 
Other than presenting the Stong-Hattori information in a convenient form, the utility of this spectral sequence is likely to be modest. However, its construction teaches the lesson: "think higher torsion-valued differentials." As an example of the value of this lesson, the key result of [15] is proved as a simple corollary to Theorem 4.3.

Proposition 5.5 (Johnson-Smith). If $X$ is a connected spectrum, then $G_{*}(X)$ is $\langle u\rangle$-torsion free if and only if hom $\operatorname{dim}_{B P_{*}} B P_{*}(X) \leq 1$.

Finally, we must justify the title of this paper; so we retrieve a version of the Stong-Hattori theorem and then show its equivalence to more conventional versions.

Theorem 6.1 (Stong-Hattori). Let $f: X \rightarrow Y$ be a map of connected spectra such that hom $\operatorname{dim}_{B P_{*}} B P_{*}(X) \leq 1$ and $G_{*}(f): G_{*}(X) \rightarrow G_{*}(Y)$ is monic; then $B P_{*}(f): B P_{*}(X) \rightarrow B P_{*}(Y)$ is monic.

Acknowledgement. The author warmly thanks Professor E. E. Floyd for his direction of the University of Virginia dissertation where much of this work first appeared [14]. He also thanks P. E. Conner, P. M. Eakin, L. Smith, R. E. Stong, and W. S. Wilson for their insight, tutorials, and helpful criticism.

2. Generalized Atiyah-Hirzebruch-Dold spectral sequences. Given a (generalized reduced) homology theory $M_{*}$ ( ) and a CW complex $X$, the Atiyah-HirzebruchDold spectral sequence is described by

$$
E_{n-q, q}^{2}(X) \cong H_{n-q}\left(X ; M_{q}\left(S^{0}\right)\right) \Rightarrow M_{*}(X) .
$$

It may be constructed from the skeletal filtration exact couple given by

$$
D_{n-q, q}^{1}(X)=M_{n}\left(X^{n-q}\right) \quad \text { and } \quad E_{n-q, q}^{1}(X)=M_{n}\left(X^{n-q} / X^{n-q-1}\right) .
$$

The derived exact couple has $D_{n-q, q}^{2}(X)=\operatorname{Image} M_{n}\left(X^{n-q-1}\right) \rightarrow M_{n}\left(X^{n-q}\right)$. Dold [11] observes that by holding $q$ fixed we get a new homology theory $M(q)_{*}$ ( ) with $M(q)_{n}(X)=D_{n-q, q}^{2}(X)$. Now suppose $R$ is a principal ideal domain and $M_{q}\left(S^{0}\right)$ is a free $R$-module. Then $H_{*}\left(X ; M_{q}\left(S^{0}\right)\right) \cong H_{*}(X ; R) \bigotimes_{R} M_{q}\left(S^{0}\right)$. The exact sequences of the derived exact couple

$$
D_{n-q, q}^{2}(X) \stackrel{i}{\longrightarrow} D_{n-q+1, q-1}^{2}(X) \stackrel{j}{\longrightarrow} E_{p, q}^{2}(X) \stackrel{k}{\longrightarrow} D_{p-1, q}^{2}(X)
$$

[19] become exact triangles of homology theories.

$$
\left[M(q)_{*}(X) \stackrel{i}{\longrightarrow} M(q-1)_{*}(X) \stackrel{j}{\longrightarrow} H_{*-q}(X ; R) \otimes_{R} M_{q}\left(S^{0}\right) \longrightarrow\right.
$$

These exact triangles show how to build spectral sequences of the form

$$
E_{*, q}^{2}(X) \cong L_{*-q}(X) \bigotimes_{R} N_{q} \Rightarrow M_{*}(X)
$$


where $L_{*}\left(\right.$ ) and $M_{*}($ ) both are generalized homology theories. The construction which follows is not the most general, but it does cover some examples from bordism theory. We have couched our description in the language of spectra, but the reader will note that all that is necessary here are exact triangles of homology theories as (2.2) satisfying analogs of (2.3) (i)-(iv).

Let $R$ be a principal ideal domain. Let $L_{*}\left(\right.$ ) and $M_{*}$ ( ) be two homology theories over $R$ induced by the (-1)-connected spectra $L$ and $M$, respectively. Also we suppose $\pi_{i}(L)$ and $\pi_{i}(M)$ to be finitely generated as $R$-modules for all integers $i$. Now suppose for each integer $q$ there is an exact triangle of spectra

$$
D(q) \stackrel{i(q)}{\longrightarrow} D(q-1) \stackrel{f(q)}{\longrightarrow} E(q) \stackrel{k(q)}{\longrightarrow} D(q)
$$

such that the following four conditions hold:

(i) The degrees of $i(q), f(q)$, and $k(q)$ are 0,0 , and -1 , respectively.

(ii) $E(q) \simeq S^{q} L \times \ldots \times S^{q} L, n(q)$ many factors of the $q$ th suspension of $L$. Here $n(q)$ is some nonnegative integer for $q$ positive. $n(0)=1$; so $E(0)=L$. For $q$ negative, $n(q)=0$; so $E(q)=*$.

(iii) $D(q)$ is $q$-connected. $D(q)=M$ for $q$ negative.

(iv) $0 \rightarrow \pi_{*}(D(q)) \stackrel{i(q) *}{\rightarrow} \pi_{*}(D(q-1)) \stackrel{f(q) *}{\rightarrow} \pi_{*}(E(q)) \rightarrow 0$ is exact.

Fix a graded free $R$-module $N_{*}$ such that the rank of $N_{q}$ is $n(q)$. In practice, we shall choose $N_{*}$ to be an $R$-polynomial ring.

Given a connected spectrum $X$, define a filtration $\left\{F_{n-q} M_{n}(X)\right\}$ of $M_{n}(X)$ $=\pi_{n}(M \wedge X)=\pi_{n}(D(-1) \wedge X)$ by

$$
F_{n-q} M_{n}(X)=\text { Image }\left\{\pi_{n}(D(q) \wedge X) \rightarrow \pi_{n}(D(-1) \wedge X)\right\}
$$

Let $X$ be $m$-connected; then $D(q) \wedge X$ is $(q+m)$-connected and our filtration is finite. (Warning: we allow $m$ to be negative!)

$$
\begin{aligned}
0 & =F_{m} M_{n}(X) \subseteq \ldots \subseteq F_{n-q} M_{n}(X) \subseteq F_{n-q+1} M_{n}(X) \subseteq \ldots \\
& \subseteq F_{n+1} M_{n}(X)=M_{n}(X)
\end{aligned}
$$

2.4. Proposition. The exact triangles described by (2.3) induce a spectral sequence $\left\{E_{n-q, q}^{r}(X), d^{r}(X)\right\}$ for any connected spectrum $X$ satisfying the following five conditions:

(i) $E_{n-q, q}^{2}(X) \cong L_{n-q}(X) \otimes_{R} N_{q}$.

(ii) $E_{n-q, q}^{\infty}(X) \cong F_{n-q+1} M_{n}(X) / F_{n-q} M_{n}(X)$.

(iii) The spectral sequence collapses if and only if $j(q)_{*}: \pi_{*}(D(q-1) \wedge X)$ $\rightarrow \pi_{*}(E(q) \wedge X)$ is epic for all $q$.

(iv) The differentials of the spectral sequence are torsion valued, i.e. $d^{r}(X)\left(E_{n-q, q}^{r}(X)\right)$ is a torsion abelian group.

(v) The spectral sequence and the above isomorphisms are natural in $X$. 
Proof. Define $D_{n-q, q}^{2}(X)=\pi_{n}(D(q) \wedge X)$ and $E_{n-q, q}^{2}(X)=\pi_{n}(E(q) \wedge X)$. The homomorphisms $i(q)_{*}, j(q)_{*}$, and $k(q)_{*}$ define a $\left(D^{2}, E^{2}\right)$ exact couple induced as (2.1). From the equivalence $E(q) \simeq S^{q} L \times \ldots \times S^{q} L$ (2.3 (iii)) where the number of factors is the same as the rank of $N_{q}$, we may fix an isomorphism $E_{n-q, q}^{2}\left(S^{0}\right) \cong L_{n-q}\left(S^{0}\right) \otimes_{R} N_{q}$ and extend this to a natural isomorphism of homology theories to fulfill (i). The naive convergence follows standardly from the fact that our filtration of $M_{n}(X)$ is finite.

$$
d^{r+1}(X): E_{n-q, q}^{n+1}(X) \rightarrow E_{n-q-r-1, q+r}^{r+1}(X)
$$

is induced by $j(q+r)_{*}\left(i(q+1)_{*} \circ \ldots \circ i(q+r-1)_{*}\right)^{-1} \circ k(q)_{*}$. So all $j(q)_{*}$ epic implies all $k(q)_{*}$ trivial implies all $d^{r+1}(X)$ trivial. Conversely if all $d^{r+1}(X)$ are trivial, then $k(q)_{*}(\pi n(E(q) \wedge X))$ is in the image of

$$
i(q+1)_{*} \circ \ldots \circ i(q+r-1)_{*}: \pi_{n-1}(D(q+r-1) \wedge X) \rightarrow \pi_{n-1}(D(q) \wedge X)
$$

for all $r$. Thus $k(q)_{*}$ is trivial and $j(q)_{*}$ is epic.

If $X_{\alpha}$ is a finite CW spectrum, $M_{n}\left(X_{\alpha}\right) \otimes \mathbf{Q}$ has a finite filtration by finitedimensional $\mathbf{Q}$-vector spaces $E_{n-q, q}^{\infty}\left(X_{\alpha}\right) \otimes \mathbf{Q}$. The usual Hurewicz-modulotorsion theorem and dimension bookkeeping arguments prove that $d^{r}\left(X_{\alpha}\right) \otimes \mathbf{Q}$ is zero. This result is extended to arbitrary connected spectra $X$ by noting that for any homology theory $J_{*}(), J_{*}(X) \cong \lim _{\rightarrow} J_{*}\left(X_{\alpha}\right)$ where the $X_{\alpha}$ range over all the finite subspectra of $X[24,12.9]$ and that $\lim _{\rightarrow}$ and $\otimes \mathbf{Q}$ are exact functors.

2.5. Corollary. Let $R$ be a principal ideal domain. Let $L$ and $M$ be $(-1)$-connected spectra inducing homology theories over $R$ with coefficient groups of finite type. Let

$$
S^{2 m} M \stackrel{\mu}{\longrightarrow} M \stackrel{\rho}{\longrightarrow} L \stackrel{\Delta}{\longrightarrow} S^{2 m} M
$$

be an exact triangle of spectra with $\mu, \rho$, and $\Delta$ having degrees 0,0 , and -1 , respectively. Suppose further that $\rho: \pi_{*}(M) \rightarrow \pi_{*}(L)$ is epic. Then there exists a natural additive spectral sequence

$$
E_{n-q, q}^{2}(X) \cong L_{n-q}(X) \otimes R\left[x_{2 m}\right] \Rightarrow M_{*}(X)
$$

with torsion-valued differentials which collapses if and only if $\rho_{*}: M_{*}(X) \rightarrow L_{*}(X)$ is epic. Here the degree of $x_{2 m}$ is $2 m$.

Proof. Define: $E(q)=*$ for $q$ negative or $q \not \equiv 0$ modulo $(2 m) ; E(q)=S^{q} L$ for $q$ nonnegative and $q \equiv 0$ modulo $(2 m) ; D(q)=M$ for $q$ negative; and $D(q-2 m)$ $=\ldots=D(q-1)=S^{q} M$ for $q$ nonnegative and $q \equiv 0$ modulo $(2 m)$.

Define $i(q)=S^{q} \mu, j(q)=S^{q} \rho$, and $k(q)=S^{q} \Delta$ for $q$ nonnegative and $q \equiv 0$ modulo $(2 m)$. Let $i(q)=$ identity and $j(q)=k(q)=*$ for $q$ otherwise. Apply (2.4). 
Examples. (1) The exact triangle $S^{2} b u_{\rightarrow}^{m_{t}} b u \stackrel{\eta}{\rightarrow} K Z \stackrel{\Delta}{\rightarrow} S^{2} b u[22]$ induces the spectral sequence

$$
E_{n-q, *}^{2}(X) \cong H_{n-q}(X) \otimes Z[t] \Rightarrow k_{*}(X)
$$

which may be identified with the conventional Atiyah-Hirzebruch-Dold one.

(2) Let $V(0)$ be a stable $Z / p Z$ Moore spectrum for an odd prime $p$. There is a map $A: S^{2 p-2} V(0) \rightarrow V(0)$ whose cofibre $V(1)$ satisfies $H^{*}(V(1) ; Z / p Z)$ $\cong E_{Z / p Z}\left[Q_{0}, Q_{1}\right]\left(Q_{0}, Q_{1}\right.$ are the first two Milnor generators of the exterior part of the $\bmod p$ Steenrod algebra). Then it is a fact that the cofibration

$$
S^{2 p-2} V(0) \stackrel{A}{\longrightarrow} V(0) \stackrel{q}{\longrightarrow} V(1)
$$

induces a short exact sequence

$$
0 \rightarrow B P_{*}\left(S^{2 p-2} V(0)\right) \stackrel{A_{*}}{\longrightarrow} B P_{*}(V(0)) \stackrel{q_{*}}{\longrightarrow} B P_{*}(V(1)) \rightarrow 0
$$

[21]. In analogy with denoting $B P_{*}(X \wedge V(0))$ as $B P_{*}(X ; Z / p Z)$, Larry Smith has suggested denoting $B P_{*}(X \wedge V(1))$ by $B P_{*}(X ; V(1))$. We then have a spectral sequence

$$
E_{s^{\prime} *}^{2}(X) \cong B P_{s}(X ; V(1)) \otimes Z_{(p)}\left[x_{2 p-2}\right] \Rightarrow B P_{*}(X ; Z / p Z)
$$

When $V(n), n>1$, exist, there are similar spectra sequences.

(3) Baas's work on bordism of manifolds with singularities [4] abounds with generalized Bockstein sequences of the form

$$
\left[M U(S)_{*}(X) \stackrel{\times\left[P_{2 n}\right]}{\longrightarrow} M U(S)_{*}(X) \rightarrow M U\left(S^{\prime}\right)_{*}(X) \longrightarrow\right.
$$

where $S$ and $S^{\prime}$ are singularity sets with $S^{\prime}=S \cup\left\{P_{2 n}\right\}$. Here $P_{2 n}$ represents a $2 n$-dimensional generator $\left[P_{2 n}\right]$ of $M U_{*}$. With indexing as in (2.5), these lead to spectral sequences

$$
E_{n-q, *}^{2}(X) \cong M U\left(S^{\prime}\right)_{n-q}(X) \otimes Z\left[x_{2 n}\right] \Rightarrow M U(S)_{*}(X)
$$

which will find use in [16].

Finally, we admit that these spectral sequences formed by (2.4) and (2.5) may not be rich in multiplicative structure. However the statement "differentials are torsion valued" can acquire new meaning. Suppose $\pi_{*}(L)$ is an integral domain and $\langle y\rangle$ the multiplicative subset generated by a nonzero element $y \in \pi_{*}(L)$. We say an element $a$ of a $\pi_{*}(L)$ module $N$ is a $\langle y\rangle$-torsion element if $y^{s} a=0$ for some positive integer $s$. The definitions of $\langle y\rangle$-torsion module and $\langle y\rangle$-torsion free module are the obvious ones. Suppose $L_{*}(X)$ is a module over $\pi_{*}(L)$, then given a spectral sequence 


$$
E_{n-q, q}^{2} \cong L_{n-q}(X) \otimes_{R} N_{q} \Rightarrow M_{*}(X)
$$

as constructed in (2.4), $E_{*, q}^{2}(X)$ has a left $\pi_{*}(L)$ multiplication. $E_{*, q}^{r}(X)$ is a quotient of a (graded) subgroup of $E_{*, q}^{2}(X)$. Fix a nonzero element $y \in \pi_{*}(L)$. If for any $z \in E_{*, q}^{2}(X)$ representing an $r$-boundary $d^{r}(X)$, there is an integer $s$ depending only on the class $d^{r}(X)$ such that $y^{s} z=0$, we say $d^{r}$ is $\langle y\rangle$-torsion valued. Note that this definition does not assume that the morphisms of the appropriate exact couple are $\pi_{*}(L)$-module homomorphisms or that $E_{*, *}^{r}(X)$ is a $\pi_{*}(L)$-module. Our experience shows that we should ask whether differentials are $\langle y\rangle$-valued where $y$ is a generator of $\pi_{*}(L)$. Affirmative answers to this query for the Stong-Hattori spectral sequence of this paper (4.4) and for the spectral sequences as in Example 3 [16] lead to Stong-Hattori type theorems.

3. The spectra $B P$ and $G$. Let $B P$ be the Brown-Peterson spectrum for the fixed prime $p$. It represents the homology theory $B P_{*}()$ constructed via the Quillen idempotent $\varepsilon: M U_{(p) *}() \rightarrow M U_{(p) *}$ ( ) [2]. $\quad H_{*}\left(B P ; Z_{(p)}\right) \cong Z_{(p)}\left[m_{1}, m_{2}, \ldots\right]$ where $m_{s}$ has degree $2\left(p^{s}-1\right)$. We identify $B P_{*}=\pi_{*}(B P)$ as a subring of $H_{*}\left(B P ; Z_{(p)}\right)$ via the Hurewicz homomorphism. $B P_{*}$ is a polynomial ring $Z_{(p)}\left[v_{1}, v_{2}, \ldots\right]$ with $v_{s}$ having degree $2\left(p^{s}-1\right)$. Liulevicius (for $p=2$ [18]) and Hazewinkel [13] have shown the generators can be defined by the formula (3.1).

$$
v_{s}=(-1)^{p+1} p m_{s}-\sum_{j=1}^{s-1} m_{s-j}\left(v_{j}\right)^{p^{s-j}} .
$$

Note that $p^{s} m_{s} \in B P_{*}$ and that $v_{1}= \pm \varepsilon[C P(p-1)]$.

We now need a few facts about $B P$ operators gathered from Zahler's exposition [25]. Let $E=\left(e_{1}, e_{2}, \ldots\right)$ be an exponent sequence of nonnegative integers, all but finitely many of which are zero. The degree of $E,|E|$, and the length of $E, n(E)$, are defined by the formulas:

$$
|E|=2 \sum_{i=1}\left(p^{i}-1\right) e_{i}, \quad n(E)=\sum_{i=1} e_{i} .
$$

We add exponent sequences termwise. If $n$ is a positive integer $n E=E+\ldots+E$ ( $n$ times). $\Delta_{i}$ is the exponent sequence, $(0, \ldots, 0,1,0, \ldots)$, where the one is in the $i$ th position. For each exponent sequence $E$, there is an operation $r_{E}$ $\in B P^{|E|}(B P)$. The action of these operators on $H_{*}\left(B P ; Z_{(p)}\right)$ is given by (3.2) and (3.3).

$$
\begin{aligned}
r_{E}\left(m_{s}\right)=m_{s-i} & \text { if } E=p^{s-i} \Delta_{i}, \\
& =0 \quad \text { if } E \neq p^{s-i} \Delta_{i}, \text { any } i \\
r_{E}(x y) & =\sum_{F+G=E} r_{F}(x) r_{G}(y) \quad \text { for } x, y \in H_{*}\left(B P ; Z_{(p)}\right) .
\end{aligned}
$$

(Here $r_{0}=$ identity where $0=(0,0, \ldots)$.)

Exponent sequences are ordered by the definition $E=\left(e_{1}, e_{2}, \ldots\right)>F$ $=\left(f_{1}, f_{2}, \ldots\right)$ if and only if: 
(i) $|E|>|F|$; or

(ii) $|E|=|F|$ and $n(E)<n(F)$; or

(iii) $|E|=|F|$ and $n(E)=n(F)$, but $e_{i}=f_{i}$ for $i>k$ and $e_{k}>f_{k}$.

As in 14.3 of [8], we have:

3.4. If $E_{1} \succeq F_{1}$ and $E_{2}>F_{2}$, then $E_{1}+E_{2}>F_{1}+F_{2}$.

3.5. Lemma. (i) $r_{\Delta_{s}}\left(v_{s}\right)=(-1)^{p+1} p$.

(ii) $r_{p \Delta_{s-1}}\left(v_{s}\right) \equiv v_{1}$ modulo $(p)$ for $s>1$.

(iii) $r_{F}\left(v_{s}\right) \equiv 0$ modulo $(p)$ for $F>p \Delta_{s-1}$.

Proof. If $|E|>2 p^{s}-2, r_{E}\left(v_{s}\right)=0=r_{E}\left(m_{s}\right)$ for dimensional reasons. This implies (i). If $E>p \Delta_{s-1}$, then $|E|>\left|p \Delta_{s-1}\right|=2\left(p^{2}-p\right)$; so $|E| \geq 2\left(p^{s}-1\right)$. (ii) and (iii) then follow by induction on degrees from (i) and (3.1)-(3.4).

For $E=\left(e_{1}, e_{2}, \ldots\right)$, define $\# E=\left(p e_{2}, p e_{3}, \ldots\right)$. Define $v^{E}$ to be the monomial $v_{1}^{e_{1}} v_{2}^{e_{2}} \ldots$ (3.3), (3.4), and (3.5) then imply:

3.6. $r_{\# E}\left(v^{E}\right) \equiv\left(v_{1}\right)^{n(E)}$ modulo $(p)$ and $r_{F}\left(v^{E}\right) \equiv 0$ modulo $(p)$ for $F \succ \# E$.

Let $k_{*}($ ) be complex connective $k$-theory. Adams has defined a splitting of $k_{(p) *}()=k_{*}() \otimes Z_{(p)}$ into a direct sum of theories $G_{*}()$ [1]. Let $G$ be the representing spectrum of $G_{*}()$. We make a pedantic separation of the properties of $G_{*}$ ( ) that we shall need.

3.7. $\pi_{*}(G) \cong Z_{(p)}[u]$ with the degree of $u$ being $2 p-2 . G_{*}(X)$ is a $Z_{(p)}[u]-$ module.

3.8. There is a map of spectra $\eta: B P \rightarrow G$ such that $\eta\left(v_{1}^{s}\right)= \pm u^{s}$ for all $s \geq 0$. If $\mu: B P \rightarrow K Z_{(p)}$ is a map of spectra inducing the edge homomorphism of the Atiyah-Hirzebruch-Dold spectral sequence, $E_{*, *}^{2} \cong H_{*}(X ; B P) \Rightarrow B P_{*}(X)$, then $\mu \simeq \eta \circ \zeta$ where $\eta$ represents a generator of $H^{0}\left(G ; Z_{(p)}\right) \cong Z_{(p)}$.

3.9. $G$ is a ring spectrum.

For an exponent sequence $E$, let $k=|\# E| /(2 p-2)=|E| /(2 p-2)-n(E)$ and let $s_{E}=\zeta \circ\left(v_{1}^{k} r_{\# E}\right) \in G^{0}(B P)$. Note that $s_{E}=s_{F}$ if and only if $E=F+t \Delta_{1}$ for some integer $t$. We define $s_{0}=\zeta \in G^{0}(B P), v^{0}=1 \in B P_{0}$, and $u^{0}=1$ $\in \pi_{0}(G)$ where 0 designates the zero exponent sequence.

3.10. Lemma (Stong [8.14.3]). Let $t$ be an integer and let $s_{E}: \pi_{t}(B P) \rightarrow \pi_{t}(G)$ designate the homomorphism induced by the element in $G^{0}(B P)$ of the same name. Then $\left\{s_{E}:|E|=t\right\}$ forms a $Z_{(p)}$-basis of $\operatorname{Hom}_{Z_{(p)}}\left(\pi_{t}(B P) ; \pi_{t}(G)\right)$.

Proof. By (3.6), (3.8), and definitions, $s_{E}\left(v^{E}\right)= \pm u^{t}$ modulo $(p),|E|=t$, and $s_{F}\left(v^{E}\right) \equiv 0$ modulo $(p)$ for $F \succ \# E$. Since $v^{E}:|E|=t$ is a free $Z_{(p)}$-basis for $\pi_{t}(B P)$, this implies $\left\{s_{E} \otimes 1:|E|=t\right\}$ is a basis of $\operatorname{Hom}_{Z_{(p)}}\left(\pi_{t}(B P) ; \pi_{t}(G)\right)$ $\otimes Z / p Z$. The lemma follows immediately from Nakayama's lemma.

Multiplication by $u \in \pi_{2 p-2}(G)$ gives a natural transformation of cohomology theories defined on finite complexes: $G^{*}() \rightarrow G^{*-2 p+2}$ ( ). By [5, Lemma 17], there is a map of spectra $m_{u}: S^{2 p-2} G \rightarrow G$ inducing this transformation. As in [22], the cofibre of $m_{u}$ is homotopy equivalent to $K Z_{(p)}$. We have the exact triangle of spectra. 
3.11. $S^{2 p-2} G_{\rightarrow}^{m_{u}} G_{\rightarrow}^{\eta} K Z_{(p)} \Delta S^{2 p-2} G$ where $\Delta$ is of degree -1 and $m_{u}, \eta$ are of degree 0 . $\eta$ is as in (3.8).

3.12. Lemma. Suppose $q=r(2 p-2) \geq 0$ and $D$ is $a(q-1)$-connected spectrum. Then any map of spectra $f: D \rightarrow G$ lifts to a map of spectra $g: D \rightarrow S^{q} G$ making (3.13) homotopy commutative.

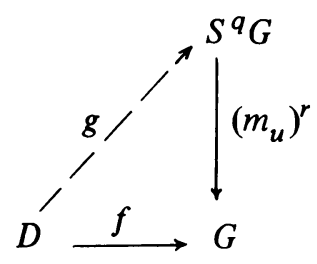

Proof. Note that $H^{i}\left(D ; Z_{(p)}\right) \cong\left[D, S^{i} K Z_{(p)}\right]=0$ for $i<q$. Apply [24, 11.10] to the mapping sequence induced by (3.11).

4. Construction of the spectral sequence. We now use our information about $\pi_{*}(B P)$ and $G$ to construct a system of exact triangles of spectra with the properties of (2.3).

4.1. Proposition. For each integer $q$, there is an exact triangle of spectra

$$
D(q) \stackrel{i(q)}{\longrightarrow} D(q-1) \stackrel{j(q)}{\longrightarrow} E(q) \stackrel{k(q)}{\longrightarrow} D(q)
$$

such that the following conditions hold:

(i) The degrees of $i(q), j(q)$, and $k(q)$ are 0,0 , and -1 , respectively.

(ii) $E(q)=S^{q} G \times \ldots \times S^{q} G$, the number of factors being $n(q)$, the rank of $\left(Z_{(p)}\left[v_{2}, v_{3}, \ldots\right]\right)_{q} \cdot($ If $n(q)=0$, we mean $E(q)=*$, the trivial spectrum.)

(iii) $D(q)$ is $q$ connected. $D(q)=B P$ for $q$ negative.

(iv) $0 \rightarrow \pi_{*}(D(q)) \stackrel{i(q)}{\rightarrow} \pi_{*}(D(q-1)) \stackrel{j(q)}{\rightarrow} * \pi_{*}(E(q)) \rightarrow 0$ is exact.

(v) Let $i(-1, q): D(q) \rightarrow D(-1)=B P$ be the composition $i(0) \circ \ldots \circ i(q)$, then diagram (4.2) commutes.

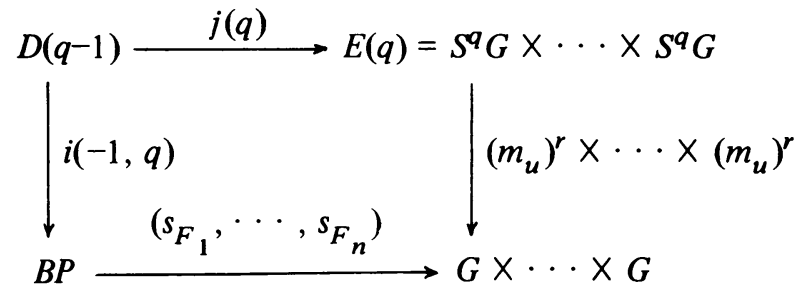

Here $r=q /(2 p-2), n=n(q)$, and $\left\{F_{1}>F_{2}>\ldots>F_{n}\right\}$ is the ordered set of exponent sequences of degree $q$ and of form $\left(0, f_{2}, f_{3}, \ldots\right)$.

Proof. We begin by defining $D(q)=B P$ and $E(q)=*$ for $q$ negative. We assume the construction is complete through the $(q-1)$ st stage and we now give the inductive construction of the $q$ th stage. 
Case 1. $g=r(2 p-2) \geq 0$. Let $F_{1}>F_{2}>\ldots>F_{n}, n=n(q)$, be as in (v). Since $D(q-1)$ is $(q-1)$-connected (by (iii) in the induction), the composition $s_{F_{i}} \circ i(-1, q)$ lifts to a map $j_{F_{i}}$ : such that (4.3) commutes (by 3.13 ).

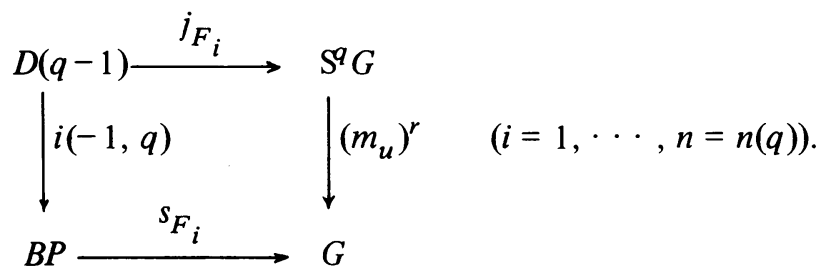

Define $j(q)=\left(j_{F_{1}}, \ldots, j_{F_{n}}\right)$. Diagram (4.2) then commutes. By [24, XI], there exists an exact triangle

$$
D(q-1) \stackrel{j(q)}{\longrightarrow} E(q) \stackrel{g}{\longrightarrow} A \stackrel{h}{\longrightarrow} D(q-1)
$$

with $g$ and $h$ having degrees -1 and 0 . Define $D(q)=A, i(q)=h$, and $k(q)=g$.

By the inductive conditions (iv) and (v), we may identify $\pi_{*}(D(q-1))$ with the intersection of the kernels of the homomorphisms $s_{F}: \pi_{*}(B P) \rightarrow \pi_{*}(G)$ for all exponent sequences $F$ with $|F|<q$. From the fact that $\pi_{*}(G) \otimes Z_{(p)}\left[v_{2}, v_{3}, \ldots\right]$ $\cong Z_{(p)}[u] \otimes Z_{(p)}\left[v_{2}, v_{3}, \ldots\right] \cong Z_{(p)}\left[v_{1}, v_{2}, v_{3}, \ldots\right]=\pi_{*}(B P)$, one can compute from inductive conditions (ii) and (iv) that $\pi_{q}(D(q-1))$ is a free $Z_{(p)}$-module of rank $n(q)$. By (3.9), $\left\{s_{F}:|F|=t\right\}$ is a basis for $\operatorname{Hom}_{Z_{(p)}}\left(\pi_{t}(B P) ; \pi_{t}(G)\right)$. Let $\left\{y^{F}\right\}$ be a basis dual to $\left\{s_{F}:|F|=t\right\}$ of

$$
\operatorname{Hom}_{Z_{(p)}}\left(\operatorname{Hom}_{Z_{(p)}}\left(\pi_{t}(B P) ; \pi_{t}(G)\right) ; \pi_{t}(G)\right) \cong \pi_{t}(B P) \text {. }
$$

Let $F_{i}^{\prime}=(t-q) /(2 p-2) \Delta_{1}+F_{i}, i=1, \ldots, n=n(q)$ where $F_{1}>\ldots>F_{n}$ were the exponent sequences as in (v) (if $t \not \equiv 0$ modulo $(2 p-2)$, everything is trivial). As $\# F_{i}^{\prime}=\# F_{i}, s_{F_{i}}^{\prime}=s_{F_{i}}$. So $s_{F_{i}}\left(y^{F_{j}^{\prime}}\right)=\delta_{i}^{j}, 1 \leq i, j \leq n$. Also $s_{F}\left(y^{F_{j}^{\prime}}\right)=0$ for exponent sequences with $|F|<q$. So $\left\{y^{F_{1}^{\prime}}, \ldots, y^{F_{n}^{\prime}}\right\}$ is a linearly independent set of elements in $\pi_{t}(D(q-1))$. $\left(m_{u}\right)^{r}: \pi_{t}\left(S^{q} G\right) \rightarrow \pi_{t}(G)$ is an isomorphism. We compute $j(q)_{*}\left(y^{F_{i}}\right)=\left(0, \ldots, 0,\left(m_{u}\right)^{r} s_{F_{i}}\left(y^{F_{i}^{\prime}}\right), 0, \ldots, 0\right), i=1, \ldots, n=n(q)$. Thus $j(q)_{*}: \pi_{*}(D(q-1)) \rightarrow \pi_{*}(E(q))$ is an epimorphism proving (iv). Then

$$
i(-1, q+1)_{*}=i(-1, q)_{*} \circ \ldots \circ i(q, q+1)_{*}: \pi_{*}(D(q)) \rightarrow \pi_{*}(B P)
$$

is a monomorphism. Since $\pi_{q}(D(q-1))$ has rank $n(q)$, we see that $D(q)$ is not only $q$-connected, but $(q+2 p-1)$-connected.

Case 2. $q=r(2 p-2)+t>0,0<t<2 p-2$. Define $E(q)=*$ and $D(q)$ $=D(q-1)=D(r(2 p-2))$.

4.4. Theorem. There is a natural spectral sequence $\left\{E_{n-q, q}^{r}(X), d^{r}(X)\right\}$ for any connected spectrum with the following five properties.

(i) $E_{n-q, q}^{2}(X) \cong G_{n-q}(X) \otimes\left(Z_{(p)}\left[v_{2}, v_{3}, \ldots\right]\right)_{q}$.

(ii) $E_{n-q, q}^{\infty}(X) \cong F_{n-q+1} B P_{n}(X) / F_{n-q} B P_{n}(X)$ where 


$$
F_{n-q} B P_{n}(X)=\text { Image }\left\{\pi_{n}(D(q) \wedge X) \rightarrow B P_{n}(X)\right\}
$$

(iii) The spectral sequence collapses if and only if $\zeta: B P_{*}(X) \rightarrow G_{*}(X)$ is epic.

(iv) The differentials of the spectral sequence are $\langle p\rangle$-torsion valued.

(v) The differentials of the spectral sequence are $\langle u\rangle$-torsion valued in the following sense. $E_{*, q}^{r}(X)$ is a quotient of a sub- $Z_{(p)}$-module of

$$
E_{*, a}^{2}(X) \cong G_{*}(X) \otimes\left(Z_{(p)}\left[v_{2}, v_{3}, \ldots\right]\right)_{q}
$$

which has a left $Z_{(p)}[u]$ multiplication. If $z \in E_{n-q-r, q+r-1}^{2}(X)$ represents $d^{r}(y)$ for $y \in E_{n-q, q}^{r}(X)$, then there is an integer $t=t(q, r)$ such that $u^{t} z=0$.

Proof. The only properties that do not follow from (2.4) are (iii) and (v). The collapsing of the spectral sequence implies $j(0)_{*}=\zeta_{X}: B P_{*}(X) \rightarrow G_{*}(X)$ is epic (2.4 (iii)). Suppose $\zeta_{X}$ is epic. By [17, Lemma 5] there is a connected spectrum $A$ and a map $f: A \rightarrow X$ such that $B P_{*}(f): B P_{*}(A) \rightarrow B P_{*}(X)$ is epic and $H_{*}(A ; Z)$ is free abelian. Since $\zeta_{X}$ is epic, the usual commutative diagram implies $G_{*}(f): G_{*}(A) \rightarrow G_{*}(X)$ is epic also. This implies $E_{*, q}^{2}(f):$ is epic in diagram (4.5). Since $H_{*}(A ; Z)$ is free abelian, $G_{*}(A)$ and $E_{*, q}^{2}(A)$ are $\langle p\rangle$-torsion free. So the spectral sequence for $A$ collapses and $j(q)_{*}(A)$ in (4.5) is epic. By commutativity $j(q)_{*}(X)$ is epic. By 2.4 (iii), the spectral sequence for $X$ also collapses,

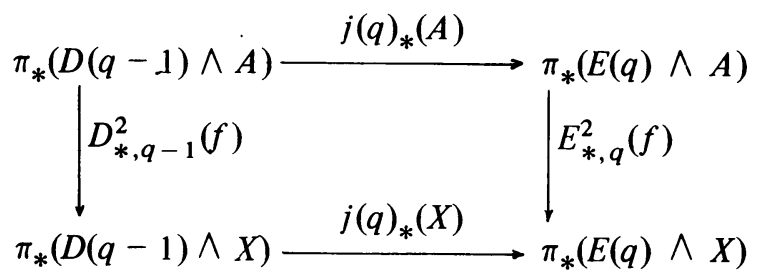

$d^{r+1}(X)$ is induced by the "composition" $\theta=j(q+r)_{*} \circ i(q, q+r)_{*}^{-1} \circ k(q)_{*}$. Diagram (4.6) commutes by (4.2) and proves that for $y \in E_{n-q, q}^{2}(X), z \in \theta(y)$, and $t=(q+r) /(2 p-2), u^{t} z=0$. This proves $(\mathrm{v})$.

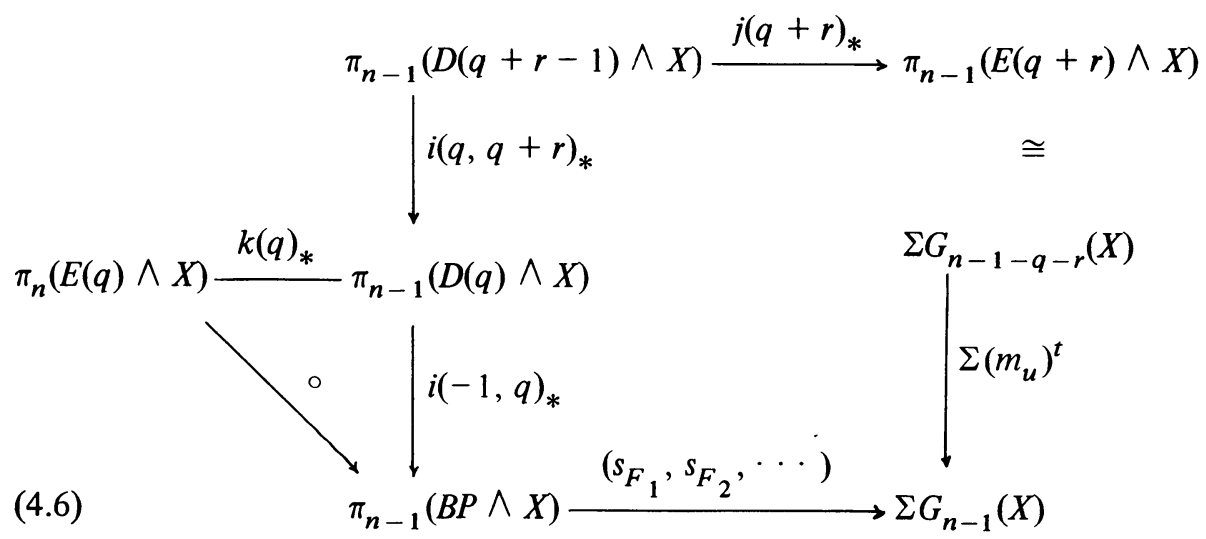


5. An application to the homological dimension of $B P_{*}$-modules. Recall from the introduction that

$$
\text { hom } \begin{aligned}
\operatorname{dim}_{B P_{*}} B P_{*}(X) & =\text { hom } \operatorname{dim}_{M U_{(p) *}} M U_{(p) *}(X) \\
& \leq \text { hom } \operatorname{dim}_{M U_{*}} M U_{*}(X) .
\end{aligned}
$$

5.1. Let $X$ be a connected spectrum. Then the Thom homomorphism $\mu$ : $B P_{*}(X) \rightarrow H_{*}\left(X ; Z_{(p)}\right)$ is an epimorphism if and only if hom $\operatorname{dim}_{B P_{*}} B P_{*}(X)$ $\leq 1$.

Proof. Adapt the proof in [9, Corollary 3.11] as in the proof of [17, Proposition 4].

5.2. Let $X$ be a connected spectrum with hom $\operatorname{dim}_{B P_{*}} B P_{*}(X)<1$; then the sequence (5.3) induced by (3.11) is short exact.

$$
0 \rightarrow G_{*}(X) \stackrel{m_{u}}{\longrightarrow} G_{*}(X) \stackrel{\eta}{\longrightarrow} H_{*}\left(X ; Z_{(p)}\right) \rightarrow 0 .
$$

Proof. We may factor $\mu$ in $(5.1)$ as the composition $B P_{*}(X) \stackrel{\zeta}{\rightarrow}(X) \stackrel{\eta}{\rightarrow}$ $H_{*}\left(X ; Z_{(p)}\right) \cdot \mu$ is epic; so $\eta$ is epic.

5.4 [15, Theorem 3]. Let $X$ be a connected spectrum such that $m_{u}: G_{*}(X)$ $\rightarrow G_{*}(X)$ is monic, then hom $\operatorname{dim}_{B P_{*}} B P_{*}(X) \leq 1$.

Proof. By the hypothesis, the sequence (5.3) is short exact and $\eta$ is epic. By 4.4 (v), the spectral sequence

$$
E_{*, *}^{2}(X)=G_{*}(X) \otimes Z_{(p)}\left[v_{2}, v_{3}, \ldots\right] \Rightarrow B P_{*}(X)
$$

must collapse. By 4.4 (iii), this implies $\zeta$ is epic. So $\mu=\eta \circ \zeta$ is epic and we apply (5.1).

5.5. Proposition (Johnson-Smith). If $X$ is a connected spectrum, then $G_{*}(X)$ is $\langle u\rangle$-torsion free if and only if hom $\operatorname{dim}_{B P_{*}} B P_{*}(X) \leq 1$.

Proof. (5.2) and (5.4).

\section{Stong-Hattori theorems.}

6.1. Stong-Hattori theorem. Let $f: X \rightarrow Y$ be a map of connected spectra such that:

(a) hom $\operatorname{dim}_{B P_{*}} B P_{*}(X) \leq 1$;

(b) $G_{*}(f): G_{*}(X) \rightarrow G_{*}(Y)$ is monic.

Then $B P_{*}(f): B P_{*}(X) \rightarrow B P_{*}(Y)$ is monic.

Proof. (a) implies that $E_{*, *}^{2}(X) \cong G_{*}(X) \otimes Z_{(p)}\left[v_{2}, v_{3}, \ldots\right]$ is $\langle u\rangle$-torsion free (5.5) and that $E_{*, *}^{2}(X)=E_{*, *}^{\infty}(X)(4.4(\mathrm{v}))$. (b) implies that

$$
E_{*, *}^{r}(f): E_{* \cdot *}^{r}(X) \rightarrow E_{* \cdot *}^{r}(Y)
$$

is monic for $r=2$. Assume $E_{*, *}^{r}(f)$ monic. Suppose $y=E_{*, *}^{2}(f)(x) \in E_{*, *}^{2}(Y)$ represents $[y]=E_{*, *}^{r}(f)(x) \in E_{*, *}^{r}$. Thus $u^{s} y \neq 0$ for all positive integers $s$. By $4.4(\mathrm{v}), y$ cannot represent any element in the image of the differentials of $d^{r}(Y)$. 
Thus the class of $y$ in $E_{*, *}^{r+1}(Y)$, which is $E_{*, *}^{r+1}(f)(x)$, is nonzero. Thus $E_{*, *}^{r+1}(f)$, is monic. By induction $E_{*, *}^{\infty}(f): E_{*, *}^{\infty}(X) \rightarrow E_{*, *}^{\infty}(y)$ is monic. By filtration induction and use of the five lemmas, $B P_{*}(f)$ is monic.

So far the only properties of $G_{*}()$ we have used are (3.7) and (3.8). We now use the ring structure of the spectrum $G(3.9)$ to justify our title for Theorem 6.1.

6.2. Proposition. Let $\operatorname{sh}_{X}: B P_{*}(X) \rightarrow G_{*}(X \wedge B P)$ be induced by the composition

$$
\begin{aligned}
& B P \wedge X \simeq B P \wedge S^{0} \wedge X \stackrel{B P \wedge i \wedge X}{\longrightarrow} B P \wedge G \wedge X \\
& \stackrel{\text { interchange }}{\longrightarrow} G \wedge X \wedge B P
\end{aligned}
$$

where $i: S^{0} \rightarrow G$ is the unit of the ring spectrum $G$. The following statements of the Stong-Hattori theorem are equivalent for a connected spectrum $X$ :

(i) hom $\operatorname{dim}_{B P_{*}} B P_{*}(X)=0$ implies $\operatorname{sh}_{X}$ is a $Z_{(p)}$-split monomorphism;

(ii) hom $\operatorname{dim}_{B P_{*}} B P_{*}(X) \leq 1$ implies $\mathrm{sh}_{X}$ is a monomorphism;

(iii) hom $\operatorname{dim}_{B P_{*}} B P_{*}(X) \leq 1$ implies $\operatorname{sh}_{X}$ is a monomorphism; spectra $f: X \rightarrow Y$ which induce monomorphisms, $G_{*}(f): G_{*}(X) \rightarrow G_{*}(Y)$ also induce monomorphisms, $B P_{*}(f): B P_{*}(X) \rightarrow B P_{*}(Y)$.

Proof. (i) implies (ii). Follow the method of [20].

(ii) implies (iii). This follows immediately from the natural equivalence

$$
G_{*}(-) \otimes_{Z_{(p)}[u]} G_{*}(B P) \rightarrow G_{*}(-\wedge B P)
$$

and the fact that $(-) \otimes_{Z_{(0)[u]}} G_{*}(B P)$ is an exact functor [20].

(iii) implies (i). Let $V(0)$ be a stable $Z / p Z$ Moore spectrum (the cofibre of a degree $\left.p \operatorname{map} S^{0} \stackrel{[p]}{\rightarrow} S^{0}\right), B P_{*}(V(0)) \cong B P_{*} / p B P_{*}$. Let $X$ be a connected spectrum with hom $\operatorname{dim}_{B P_{*}} B P_{*}(X)=0$, then hom $\operatorname{dim}_{B P_{*}} B P_{*}(X \wedge V(0))=1$ (a specific $B P_{*}$ resolution is induced by $X \wedge S^{0} \stackrel{X \wedge[p]}{\rightarrow} X \wedge S^{0} \rightarrow X \wedge V(0)$ ). Let $\mu: G \wedge G$ $\rightarrow G$ be the multiplication of the ring spectrum $G$. Then $\mu \circ i \simeq G$ and $G_{*}(i \wedge W): G_{*}(W) \rightarrow G_{*}(G \wedge W)$ has left inverse $(\mu \wedge W)_{*}$. So it follows from (iii) that $B P_{*}(i \wedge W): B P_{*}(W) \rightarrow B P_{*}(G \wedge W)$ is monic for $W=X$ or $X \wedge V(0)$. Let

$$
\phi_{W}: B P_{*}(W) \otimes Z / p Z=B P_{*}(W) \otimes_{B P_{*}} B P_{*}(V(0)) \rightarrow B P_{*}(W \wedge V(0))
$$

be induced by the Künneth pairing. By $[9,8.4], \phi_{X}$ is a monomorphism (prove this directly using the $B P_{*}$ resolution of $V(0)$ induced by $\left.S^{0} \stackrel{[p]}{\rightarrow} S^{0} \rightarrow V(0)\right)$. In the commuting diagram (6.3), $\phi_{X}$ and $(i \wedge X \wedge V(0))_{*}$ are monic; so $(i \wedge X)_{*} \otimes Z / p Z$ is also. Since $B P_{*}(X)$, is $\langle p\rangle$-torsion free and $(i \wedge X)_{*}$ is monic, this implies $(i \wedge X)_{*}$ is $Z_{(p)}$-split monic. The interchange map in the definition of $\mathrm{sh}_{X}$ is an equivalence; so $\mathrm{sh}_{X}$ is split monic. 


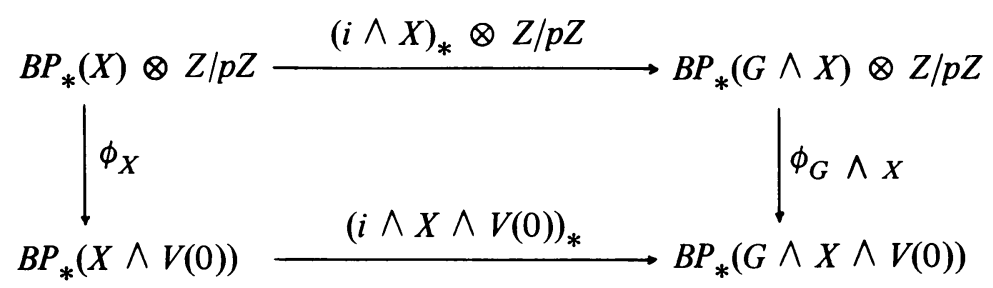

Larry Smith has shown that the Stong-Hattori homomorphism fails to be monic for the spectrum $V(1)$ which realizes the $\bmod p$ Steenrod algebra module $E_{Z / p Z}\left[Q_{0}, Q_{1}\right], p$ odd. (See [24, Example 2, §2].) Here is another example. Conner and Smith build stable complexes

$$
X(\eta)=S^{0} \cup_{2} e^{1} \cup_{\bar{\eta}} e^{3} \quad \text { and } X(\eta, \nu)=S^{0} \cup_{2} e^{\prime} \cup_{\bar{\eta}} e^{3} \cup_{\bar{\nu}} e^{7}
$$

using the first two elements of Hopf invariant one, $\eta \in \pi_{1}^{s}$ and $\nu \in \pi_{3}^{s}$ [10]. They are the simplest complexes with

$$
\text { hom } \operatorname{dim}_{M U_{*}} M U_{*}(X(\eta))=\text { hom } \operatorname{dim}_{M U_{(2) *}} M U_{(2) *}(X(\eta))=2
$$

and

$$
\text { hom } \operatorname{dim}_{M U_{*}} M U_{*}(X(\eta, \nu))=\text { hom } \operatorname{dim}_{M U_{(2) *}} M U_{(2) *}(X(\eta, \nu))=3,
$$

respectively. There is a cofibration $X(\eta) \stackrel{i}{\rightarrow} X(\eta, \nu) \rightarrow S^{7}$ which clearly induces a monomorphism on $Z_{(2)}$ homology. So for $G_{*}()=k_{*}() \otimes Z_{(2)}$

$$
G_{*+1}\left(S^{7}\right) \stackrel{\partial}{\longrightarrow} G_{*}(X(\eta)) \stackrel{G_{*}(i)}{\longrightarrow} G_{*}(X(\eta, \nu)),
$$

$G_{*}(i)$ will be monic modulo $\langle 2\rangle$-torsion. Since hom $\operatorname{dim}_{M U_{(2) *}} M U_{(2) *}(X(\eta))>1$, we cannot have a short exact sequence as (5.3) else we would contradict (5.4).

$$
H_{j}\left(X(\eta) ; Z_{(p)}\right) \stackrel{\Delta}{\longrightarrow} G_{j-3}(X(\eta)) \stackrel{m_{u}}{\longrightarrow} G_{j-1}(X(\eta)) .
$$

So $\Delta$ must be nonzero in (6.5) for some $j$. The only possibility is when $j=3$. We compute that $G_{0}(X(\eta)) \cong Z / 2 Z$ is the only torsion group in $G_{*}(X(\eta))$. As $\partial\left(G_{1}\left(S^{7}\right)\right)=0$ in $(6.5), G_{*}(i)$ must be monic. If $B P_{*}(i)$ were monic, we would have a short exact sequence

$$
0 \rightarrow B P_{*}(X(\eta)) \stackrel{B P_{*}(i)}{\longrightarrow} B P_{*}(X(\eta, \nu)) \rightarrow B P_{*}\left(S^{7}\right) \rightarrow 0
$$

with $B P_{*}$ modules with homological dimensions 2,3 , and 0 respectively. This disagrees with [7, VI, 2.4]; we conclude that $\operatorname{sh}_{X(\eta)}$ cannot be monic.

\section{REFERENCES}

1. J. F. Adams, Lectures on generalized cohomology, Category Theory, Homology Theory andTheir Applications, III (Battelle Inst. Conf., Seattle, Wash., 1968), Springer, Berlin, 1969, pp. 1-138. MR 40 \#4943. 
2. J. F. Adams, Quillen's work on formal groups and complex cobordism, Lecture Notes, University of Chicago, Chicago, Ill., 1970.

3. J. F. Adams and A. Liulevicius, The Hurewicz homomorphism MU and BP, University of Chicago, Chicago, Ill., 1970 (preprint).

4. N. A. Baas, On bordism theory of manifolds with singularities, Preprint series $1969 / 70$, no. 31, Aarhus Universitet, Aarhus.

5. E. H. Brown, Jr., Cohomology theories, Ann. of Math. (2) 75 (1962), 467-484. MR 25 \# 1551.

6. E. H. Brown, Jr. and E. P. Peterson, $A$ spectrum whose $Z_{p}$ cohomology is the algebra of reduced pth powers, Topology 5 (1966), 149-154. MR 33 \# 719.

7. H. Cartan and S. Eilenberg, Homological algebra, Princeton Univ. Press, Princeton, N.J., 1956. MR 17, 1040.

8. P. E. Conner and E. E. Floyd, The relation of cobordism to K-theories, Lecture Notes in Math., no. 28, Springer-Verlag, Berlin and New York, 1966. MR 35 \#7344.

9. P. E. Conner and L. Smith, On the complex bordism of finite complexes, Inst. Hautes Études Sci. Publ. Math. No. 37 (1969), 117-221. MR 42 \#2473.

10. - On the complex bordism of finite complexes. II, J. Differential Geometry 6 (1971), 135-174.

11. A. Dold, On general cohomology. Chaps.1-9, Lectures given at the Nordic Summer School in Math. (1968), Matematisk Institut, Aarhus Universitet, Aarhus, 1968. MR 40 \# 8045.

12. A. Hattori, Integral characteristic numbers for weakly almost complex manifolds, Topology 5 (1966), 259-280. MR 33 \#742.

13. M. Hazewinkel, Constructing formal groups. I. Over $Z_{(p)}$-algebras, Report of the Econometric Institute \#7119, Netherlands School of Economics, 1971.

14. D. C. Johnson, Cobordism and connective K-theory, Dissertation, University of Virginia, Charlottesville, Va., 1970.

15. D. C. Johnson and L. Smith, On the relation of complex cobordism to connective K-theory, Proc. Cambridge Philos. Soc. 70 (1971), 19-22.

16. D. C. Johnson and W. S. Wilson, Projective dimension and Brown-Peterson homology, University of Kentucky, Lexington, Ky., 1972 (preprint).

17. P. S. Landweber, Complex bordism of classifying spaces, Proc. Amer. Math. Soc. 27 (1971), 175-179. MR 42 \#3782.

18. A. Liulevicius, On the algebra $B P_{*}(B P)$, University of Chicago, Chicago, Ill., 1971 (preprint).

19. S. Mac Lane, Homology, Die Grundlehren der math. Wissenschaften, Band 114, Academic Press, New York; Springer-Verlag, Berlin, 1963. MR 28 \# 122.

20. L. Smith, A note on the Hattori-Stong Theorem, Illinois J. Math. (to appear).

21._ On realizing complex bordism modules. Applications to the stable homotopy of spheres, Amer. J. Math. 92 (1970), 793-856. MR 43 \# 1186a.

22._- On the relation of connective K-theory to homology, Proc. Cambridge Philos. Soc. 68 (1970), 637-639. MR 41 \#7677.

23. R. E. Stong, Relations among characteristic numbers. I, Topology 4 (1965), 267-281. MR 33 \#740.

24. R. Vogt, Boardman's stable homotopy category, Lecture Notes Series, no. 21, Matematisk Institut, Aarhus Universitet, Aarhus, 1970. MR 43 \#1187.

25. R. Zahler, The Adams-Novikov spectral sequence for the spheres, Ann. of Math. (2) 96 (1972), 480-504.

Department of Mathematics, University of Kentucky, LeXington, KentuCky 40506. 